\title{
Intra-articular Adenosine, Lidocaine and Magnesium (ALM) solution decreases postoperative joint fibrosis in an experimental knee implant model
}

Jodie L. Morris ${ }^{1,2}$, Hayley L. Letson ${ }^{2}$, Peter McEwen ${ }^{1}$, Erik Biros ${ }^{2}$, Constantin Dlaska ${ }^{1}$, Kaushik Hazratwala ${ }^{1}$, Matthew Wilkinson ${ }^{1}$ and Geoffrey P. Dobson ${ }^{2^{*}}$ (D)

\begin{abstract}
Background: There is currently no drug therapy to prevent arthrofibrosis following knee surgery. We aimed to determine if the anti-ischemic and anti-inflammatory drug adenosine, lidocaine and $\mathrm{Mg}^{2+}(\mathrm{ALM})$, reduces surgeryrelated arthrofibrosis in a rat model of knee implant surgery.

Methods: Male Sprague-Dawley rats $(n=24)$ were randomly divided into ALM or saline groups. The right knee of each animal was implanted with custom titanium (femur) and polyethylene (tibia) implants, and the left knee served as a non-operated control. An intra-articular ALM or saline bolus $(0.1 \mathrm{ml})$ was administered at the end of surgery, and animals monitored for 4 weeks. Fibrotic changes were assessed by macroscopic examination, histopathology, and expression of key inflammatory and fibrotic markers in the joint capsule and infrapatellar fat pad (IFP).

Results: Knee swelling was evident in both groups at 4 weeks. However, range of motion was 2-fold higher in the ALM-treated knees, and differences in macroscopic pathology indicated improved healing, compared to the control group. Histologically, ALM treatment also led to significantly decreased synovitis and fibrotic pathology in the joint capsule and IFP compared to saline controls. RNA and protein expression profiles of pro-fibrotic mediators (a-SMA, TGF- $\beta 1$, FGF1, PDGFA) were also significantly lower in knees from ALM-treated animals. In addition, the expression of inflammatory mediators was lower in plasma (IL-1 $\beta, I L-10)$ and joint tissue (NFKB, IL-1 $\beta, I L-12), 4$ weeks after surgery.

Conclusion: We show that intra-articular administration of a single ALM bolus significantly decreased fibrotic pathology and synovitis in an experimental model of knee implant surgery, by blunting inflammation and modulating essential genes of fibrosis. ALM has the therapeutic potential for translation into humans undergoing knee replacement surgery.
\end{abstract}

Keywords: Total knee arthroplasty, Stiffness, Arthrofibrosis, Inflammation

\footnotetext{
* Correspondence: geoffrey.dobson@jcu.edu.au

${ }^{2}$ Heart, Trauma and Sepsis Research Laboratory, College of Medicine and

Dentistry, James Cook University, Townsville, Queensland 4811, Australia

Full list of author information is available at the end of the article
}

(c) The Author(s). 2021 Open Access This article is licensed under a Creative Commons Attribution 4.0 International License, which permits use, sharing, adaptation, distribution and reproduction in any medium or format, as long as you give appropriate credit to the original author(s) and the source, provide a link to the Creative Commons licence, and indicate if changes were made. The images or other third party material in this article are included in the article's Creative Commons licence, unless indicated otherwise in a credit line to the material. If material is not included in the article's Creative Commons licence and your intended use is not permitted by statutory regulation or exceeds the permitted use, you will need to obtain permission directly from the copyright holder. To view a copy of this licence, visit http://creativecommons.org/licenses/by/4.0/. 


\section{Background}

Stiffness and arthrofibrosis are common fibrotic joint complications following knee surgery $[1,2]$. Acquired idiopathic stiffness accounts for up to $58 \%$ of repeat interventions following primary total knee arthroplasty (TKA) [3], and arthrofibrosis is responsible for up to $28 \%$ of 90 -day hospital readmissions and $10 \%$ of revision surgeries within the first 5 years of surgery [1, 4]. PostTKA arthrofibrosis reflects a more severe fibrotic pathology and restriction in joint function, with $\sim 25 \%$ of these requiring additional surgery and manipulation to remove the adhesions and scar tissue $[1,4]$.

The key drivers of postoperative joint stiffness and fibrosis are believed to be excessive inflammation, the proliferation of metaplastic fibroblasts, increased extracellular matrix (ECM) proteins, and fibrous scar tissue that leads to restricted joint motion and pain $[1,2,5]$. These secondary complications arise primarily from the trauma of surgery and involve the synovial membrane, extracapsular structures (e.g. medial and lateral ligaments) and the infrapatellar fat pad (IFP) [1, 2, 4-7]. Despite aggressive physical therapy regimens combined with anti-inflammatory medications, there are no effective drugs that prevent stiffness or arthrofibrosis following knee surgery. Previously, we have shown that intravenous administration of adenosine, lidocaine and $\mathrm{Mg}^{2+}$ (ALM) therapy reduces inflammation and secondary injury progression following hemorrhagic shock, traumatic brain injury, and major surgery in rat and porcine models [8-14]. ALM has also been shown to improve cell viability of human chondrocytes compared to saline alone [15]. Based on ALM's anti-inflammatory, anti-ischemic, and coagulopathy correction properties, we hypothesized that an intra-articular bolus administered before skin closure may reduce arthrofibrosis in a rat model of knee implant surgery.

\section{Methods}

\section{Study design and surgical procedure}

Conventional, twenty-week-old male Sprague-Dawley rats (350 to $450 \mathrm{~g}$ ) were used. Animals were individually housed in ventilated cages (Tecniplast ${ }^{\oplus}$ Australia, NSW, Australia) in a 14-10 h light-dark cycle under controlled temperature $\left(21-22^{\circ} \mathrm{C}\right)$ and humidity $(65-75 \%)$ conditions, with access to standard rodent pellets (Specialty Feeds, WA, Australia) and water ad libitum. Using a random number generator (Research Randomizer.org) animals were randomly allocated to treatment groups, and within each treatment group, whether end-point tissue samples were to be fixed (for microCT and histology, $n=6$ per group) or snap-frozen in liquid nitrogen (gene and protein expression, $n=6$ per group). After a 7 -day acclimation period, knee implant surgery was performed on rats $(n=24)$ under isoflurane anesthesia using surgical techniques and materials described previously [16]. Surgeries were performed on 8 animals per surgery day, with equal numbers of animals per treatment group and randomization of the order of animals undergoing surgery.

Three trained orthopedic surgeons contributed to this study, with surgeons blinded to treatment groups and surgeries conducted according to a standard protocol. Surgeon contribution was equivalent across control and treatment groups, with surgery time and blood loss comparable for both groups (Table S1). Briefly, a UHXLPE implant was seated in a small mantle of gentamicinladen bone cement (Heraeus Palacos ${ }^{\oplus} \mathrm{R}+\mathrm{G}$, Zimmer Biomet, Sydney, Australia) within the proximal tibia. A sterile titanium implant was press-fit into a defect created in the distal femur. Following implantation, mild abrasion of the synovium (medial and lateral gutters, suprapatellar pouch, and retro patellar fat pad) was performed using sterile dressing forceps, with a standardized protocol of two passes each across each femoral condyle and the medial and lateral tibial plateaus. Following patella repositioning, the capsule and skin were closed with PDS II 5-0 (Ethicon, New Jersey, United States of America) and monocryl 5-0 (Ethicon), respectively. A continuous subcuticular technique was used for skin closure and the surgical site swabbed with povidone-iodine solution, then sprayed with OpSite ${ }^{\mathrm{rm}}$.

Immediately after skin closure and prior to recovery from anesthesia, animals received pre-emptive analgesic consisting of a $0.05 \mathrm{mg} / \mathrm{kg}$ subcutaneous injection of buprenorphine (Temgesic ${ }^{\circ}$ ) in a $1 \mathrm{~mL}$ bolus of saline. Postoperative analgesia (buprenorphine, 0.01-0.05 mg/ $\mathrm{kg}$ ) was administered at 6 and $12 \mathrm{~h}$ post-surgery, with analgesic administered 12-hourly thereafter, according to pain scores of individual animals. Clinical signs including body weight, temperature and weight-bearing activity were monitored daily throughout the experimental period. Animals were sacrificed 4 weeks post-surgery with an overdose of pentobarbital $(100 \mathrm{mg} / \mathrm{kg})$ for gross pathology, molecular and histological evaluation.

\section{Treatment}

Adenosine (A9251), Lidocaine- $\mathrm{HCl}$ (L5647), and $\mathrm{MgSO}_{4}$ (M7506) were purchased from Sigma-Aldrich. Animals received an intra-articular (IA) bolus of ALM $(0.1 \mathrm{ml} ; 1$ $\mathrm{mM}$ adenosine, $3 \mathrm{mM}$ lidocaine, and $2.5 \mathrm{mM} \mathrm{MgSO}_{4}$ in $0.9 \% \mathrm{NaCl}$ ) immediately following capsule closure and prior to skin closure [8]. Control animals received an IA bolus of $0.9 \% \mathrm{NaCl}(0.1 \mathrm{ml})$ prior to skin closure.

\section{Hematology, ROTEM and inflammatory assessments}

Blood was collected under anesthesia via the lateral tail vein during the experimental period or via terminal cardiac puncture at 4 weeks post-surgery (Fig. S1). 
Complete blood cell examination was carried out using a VetScan HM5 hematology analyzer (Abaxis, CA, USA). Rotational thromboelastometry (ROTEM; Tem International, Munich, Germany) was conducted on whole blood according to manufacturer's instructions. Three 60-min assays were performed simultaneously for each sample: EXTEM (extrinsically activated test using tissue factor), INTEM (intrinsically activated test using ellagic acid), and FIBTEM (EXTEM-activated test with cytochalasin D to inhibit platelet contribution to clot formation). Parameters include clotting time (CT; seconds); alpha angle $\left(\alpha^{\circ}\right)$; clot amplitude $(\mathrm{mm})$; maximum clot firmness (MCF, mm); and maximum lysis (\%).

Blood samples were centrifuged and plasma collected and stored at $-80{ }^{\circ} \mathrm{C}$ until further analysis. Vascular injury markers (adiponectin, sE-selectin, sICAM-1, von Willebrand Factor) were measured in plasma using Milliplex ${ }^{\odot}$ Rat Vascular Injury Magnetic Bead Panel 2 (Abacus ALS, Meadowbrook, Queensland), in combination with the Magpix ${ }^{\circledR}$ analyzer (Luminex Corporation, Austin, Texas, USA). Inflammatory chemokines and cytokines (MCP-1, TNF- $\alpha$, IL-1 $\beta$, IL-6, IL-12p70, IFN- $\gamma$, IL-4, IL-10, TGF- $\beta 1$ ) were measured in plasma and joint tissue homogenates using Milliplex ${ }^{\odot}$ Rat Cytokine/Chemokine Magnetic Bead Panel (Abacus ALS), and Rat Transforming Growth Factor- $\beta 1$ (TGF- $\beta 1$ ) ELISA (CusaBio). Assays were performed according to manufacturer instructions, with samples measured in duplicate. Inflammatory markers in joint capsular tissue samples were normalized to total protein (Pierce BCA protein assay, ThermoFisher Scientific). Detection ranges for analytes were $29.3-120,000 \mathrm{pg} / \mathrm{ml}$ for MCP-1, 15.3-60, $000 \mathrm{pg} / \mathrm{ml}$ for IFN- $\gamma, 2.4-10,000 \mathrm{pg} / \mathrm{ml}$ for IL- $1 \beta$ and TNF- $\alpha, 73.2-300,000 \mathrm{pg} / \mathrm{ml}$ for IL-6, $12.2-50,000 \mathrm{pg} / \mathrm{ml}$ for IL-12p70, 4.9-20,000 pg/ml for IL-4, 7.3-30,000 pg/ $\mathrm{ml}$ for IL-10, $6.25-400 \mathrm{pg} / \mathrm{ml}$ for TGF- $\beta, 0.1-40 \mathrm{ng} / \mathrm{ml}$ for sICAM-1, $0.1-50 \mathrm{ng} / \mathrm{ml}$ for sE-selectin, $0.3-250 \mathrm{ng}$ / $\mathrm{ml}$ for adiponectin, and $0.7-500 \mathrm{ng} / \mathrm{ml}$ for $\mathrm{vWF}$.

\section{Joint angle measurement}

The extension angle between the longitudinal axis of the femur and the tibia was measured in triplicate using an angulometer with $20 \mathrm{~g}$ force for both the right (operated) and left (non-operated, control) knee [17]. Data are expressed as the mean extension angle for control and operated knees from saline- and ALM-treated animals.

\section{Tissue harvest}

Following blood collection at 4 weeks post-surgery, animals within each treatment arm were sacrificed to assess joint tissue metrics. Macroscopic morphological analysis of the intact joint, the articular surfaces, capsular and synovial tissue was performed using a modified grading system (Table S2) [18], with scores ranging from 0 (healthy, non-operated knee) to 9 (severe knee pathology). Knees were graded independently by two blinded scorers and scores averaged. Agreement between scorers was excellent, with an intraclass correlation coefficient of 0.7 (95\% CI 0.40 to 0.86 ), and the mean difference in the score being 0.02 (95\% CI, -0.13 to 0.17 ).

Following evaluation of joint range of motion and gross pathology, animals were perfused with ice-cold 0.1 $\mathrm{M}$ phosphate-buffered saline (PBS, pH 7.4). The operated (right) and non-operated control (left) knee were dissected through a medial parapatellar approach using a surgical blade (size 22). Anteromedial capsular tissue (Fig. 1c) was separated from superficial tendons under a dissection microscope, snap-frozen and stored at $-80{ }^{\circ} \mathrm{C}$ for subsequent RNA/protein extraction and analysis. After removal of the patella and anteromedial capsule, knees were fixed in $4 \%$ paraformaldehyde (PFA) for routine histology.

\section{RNA isolation, cDNA synthesis, and real-time polymerase chain reaction}

Quantitative real-time reverse transcription polymerase chain reaction (qPCR) was used to assess relative expression of selected genes involved in inflammation and fibrosis within joint capsule tissue. Briefly, snap-frozen tissue $(20 \mathrm{mg}$ ) was homogenised in $1 \mathrm{ml}$ Trizol (Qiagen) using disposable mortar and pestle. Total RNA was isolated from dissociated tissue samples using the RNeasy mini kit (Qiagen) according to manufacturer's instructions. Primers were designed using the AlleleID software (PREMIER Biosoft), and manufactured and purchased from Merk (Table S3). The selection includes alphasmooth muscle actin-2 (Acta2), collagens (Col1a1, Col3a1), matricellular proteins $(\mathrm{Ccn} 2)$, growth factors (Tgfb1, Fgf1, Pdgfa), metalloproteinases (Mmp3, Mmp13), xylosyltransferase (Xylt1) and inflammatory mediators (Nos2, Il1b, Il6). The relative expression of each gene was calculated using the concentration-Ctstandard curve method and normalized using the average expression of the housekeeping ribosomal protein S13 (Rps13) gene for each sample using the Rotor-Gene Q operating software version 2.0.24 (Qiagen). Rps13 was chosen as the "housekeeping" gene since previous work has shown its expression to be similar across rat tissue types [14]. A one-step QuantiTect SYBR Green RT-PCR Kit (Qiagen) was used in combination with the QuantiTect Primer Assays (Qiagen) according to manufacturer's instructions, with $20 \mathrm{ng}$ of total RNA as template.

\section{Histomorphometry}

For routine histology, fixed intact knee joints were decalcified (14\% EDTA), processed and paraffin-embedded. Consistent with OARSI recommendations, sections $(4 \mu \mathrm{m})$ were cut in the frontal plane, spaced at $50 \mu \mathrm{m}$ 

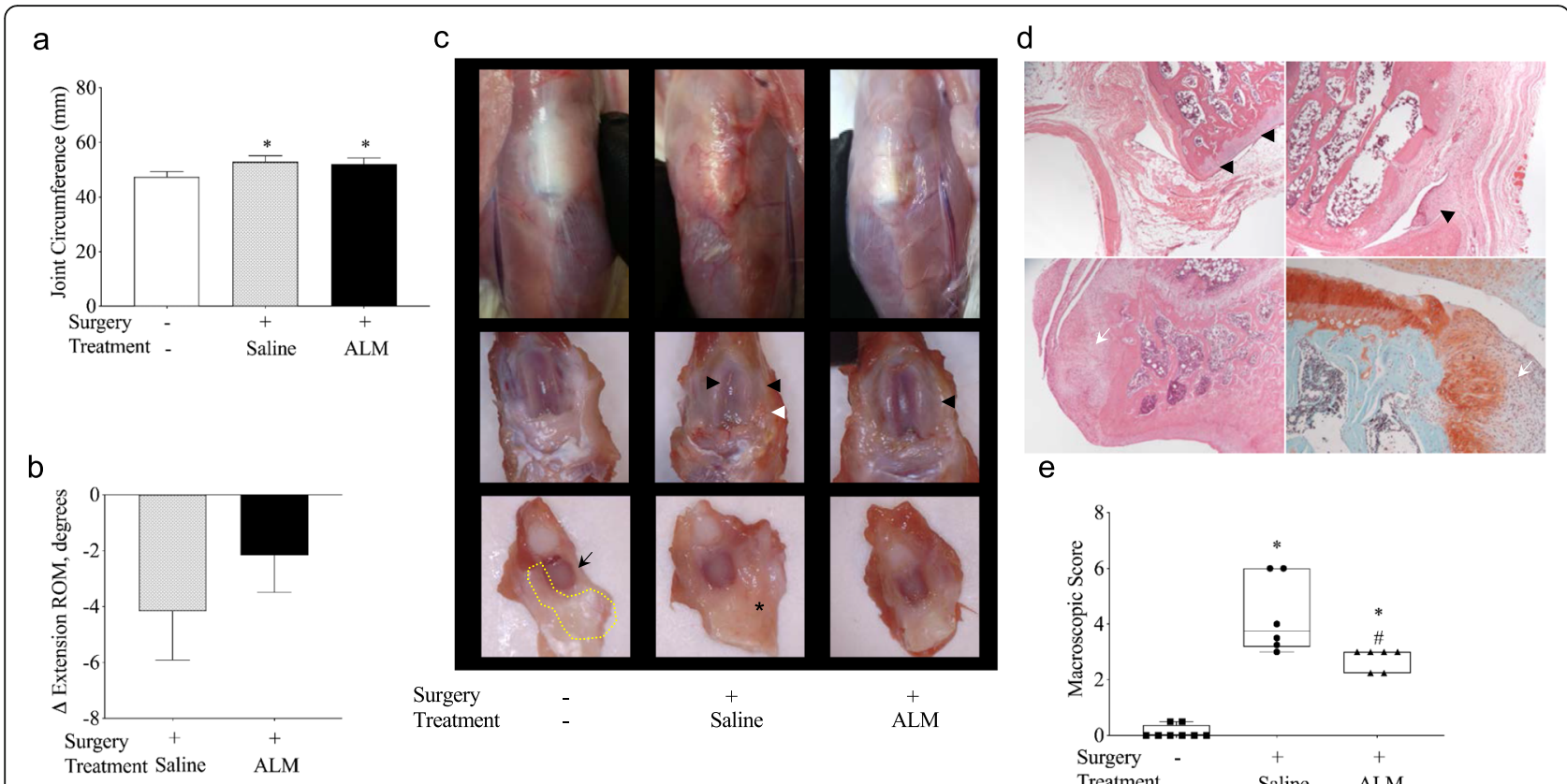

e

Fig. 1 Macroscopic changes in knees at 4 weeks postoperative for animals that received an intra-articular bolus of ALM or saline immediately following knee implant surgery, and prior to skin closure. a Compared to non-operated control knees, the circumference of implanted knees was increased significantly for saline- and ALM-treated animals. b At 4 weeks post-surgery, the difference in the range of motion (ROM) in extension for operated and non-operated knees tended to be greater for saline- compared to ALM-treated animals, though this difference was not statistically significant. c Representative images of a non-operated knee, and knees from saline-treated and ALM-treated animals at 4 weeks postsurgery. External views of the knee joints after removal of the skin (upper panel). Internal views of the joints following dissection (middle, lower panels). Arrow indicates the location of the patella. Synovial thickening (asterisk), adhesions (white arrowhead) and articular cartilage roughness (black arrowheads) were evident in operated knees. The yellow dotted line indicates the anteromedial region of capsular tissue dissected for subsequent molecular analyses. d Adhesion of the synovium and femoral condyles was evident histologically (arrowheads, upper panels). Macroscopic changes to articular surfaces corresponded to granulation tissue, fibrocartilage and subchondral bone remodeling (white arrows) apparent on the femur (lower left panel) and tibia (lower right panel). e Macroscopic pathology scores were significantly reduced for the operated joints that were administered intra-articular ALM, compared to saline controls. Data shows mean $\pm \mathrm{SD}$. ${ }^{*} P<0.05$, vs. baseline (no surgery, no treatment), $\# P<0.05$, compared to saline controls; $n=12$ per group (circumference, ROM), $n=6$ per group (macroscopic scoring)

intervals and spanning the entire knee joint [19]. Stained sections were visualized with light microscopy (Nikon Eclipse i50; Japan) and digitized for histomorphometric analysis using Image ${ }^{\circ}$ software (v1.52p; National Institutes of Health, EUA).

Medial capsule thickness was measured in hematoxylin and eosin (H\&E) stained sections (40x magnification), with the mean capsule thickness of each animal determined from two sections and five individual measurements per section. Semi-quantitative evaluation of synovitis and fibrotic changes in the IFP was performed by a blinded investigator on $\mathrm{H} \& \mathrm{E}$-stained sections (40x magnification) and according to the scoring system adapted from Takahashi et al. [20] and Kitagawa et al. [21] (Table S4). Masson-Goldner trichrome staining was used to assess collagen density in the IFP. The mean percentage of the green-stained area (indicating collagen fibres) of trichrome-stained sections in comparison to the total area was calculated using the Trainable Weka Segmentation plugin in ImageJ.
For immunohistochemical analysis of $\alpha$-SMA expression in capsular tissue and IFP, heat-mediated antigen retrieval was performed in citrate buffer ( $\mathrm{pH} 6$; Sigma Aldrich, North Ryde, Australia) for $20 \mathrm{~min}$ at $95^{\circ} \mathrm{C}$. Endogenous peroxidase and phosphatase were blocked with BLOXALL blocking solution (Vector Laboratories, Burlingame, USA), and non-specific binding with Animal-Free Blocker (Vector Labs). Sections were incubated overnight at $4{ }^{\circ} \mathrm{C}$ with mouse anti-alpha smooth muscle actin ( $\alpha$-SMA) antibody at 1:1000 dilution (1A4; Abcam). A biotinylated secondary antibody (horse antimouse/rat IgG; Vector Labs) was applied for $30 \mathrm{~min}$ at room temperature, followed by detection with an avidinbiotin-based peroxidase kit (ABC Elite; Vector Labs). Immunoreactivity was visualized with 3,3'-diaminobenzidine staining and sections counterstained with hematoxylin. Semi-quantitative assessment of positive staining for $\alpha$ SMA was carried out by a blinded investigator on five randomly selected high-powered fields (400x magnification) per sections and two sections per joint. 


\section{Statistics}

Sample sizes $(\mathrm{n})$ were determined from a priori power analysis using G-power3 program (Heinrich-Heine-Universität Düsseldorf) using Acta2 gene expression as the outcome measure with effect size Cohen's d $=1.65$ (Critical $\mathrm{t}=1.96 ; \mathrm{df}=11$; sample size $=6 ; \alpha=0.05$; calculated power $=0.84)$. Statistical analyses were performed using GraphPad Prism for Mac software (version 7). Data normality was assessed using the Shapiro-Wilks test, with Levene's test used to determine equality of variances. Independent samples t-tests were used for between-groups comparison for normally distributed data (hematology parameters, inflammatory cytokine concentrations). Non-normally distributed data (joint angle, macroscopic and histological scores, percentage positive $\alpha$-SMA staining) were compared using a Mann-Whitney U test. MILLIPLEX Analyst 5.1 software (Luminex Corporation, Austin, Texas, USA) was used to determine cytokine and chemokine concentrations with a 5-parametric logistic weighted curve fit. Results are expressed as mean \pm standard deviation (SD), with significance set at $P<0.05$.

\section{Results}

\section{Operative and postoperative metrics}

The time duration for knee implant surgeries, total time under anesthesia and blood loss was comparable for animals in saline- and ALM-treated groups (Table S1). All animals recovered from anesthesia and were conscious and ambulant within $15 \mathrm{~min}$. No animals showed signs of infection, nor were there any deaths during the experimental period. Minor weight loss occurred in the first week post-surgery; however, all animals had returned to pre-surgical body weights by 4 weeks postsurgery (Table S5).

\section{Gross inspection of macroscopic changes in the joint}

Four weeks after surgery, animals were euthanized and gross inspection of macroscopic changes in operated knees was performed following removal of skin. Compared to non-operated control knees, operated knees were visibly enlarged at 4 weeks post-surgery, with knee joint circumference increased 1.1 -fold $(P<0.001$; Fig. 1a). However, the joint circumference was comparable for operated knees that were administered saline or ALM intraoperatively $(P=0.396$; Fig. 1a). Similarly, the extension ROM of operated knees was lower than that of native knees. Compared to non-operated knees, a mean decrease of 4.2 and 2.2 degrees, respectively was observed in extension ROM for saline-treated and ALMtreated knees 4 weeks following surgery $(P=0.37$; Fig. 1b). Upon joint dissection it was evident that increases in the joint size of operated knees was associated with expansion of the synovium and joint capsule, with adhesions apparent in the operated knees (Fig. 1c). Mild-to- moderate pathological changes were also evident on the articular surfaces of the distal femur and proximal tibia in operated knees, compared to non-operated control knees (Fig. 1c). Histologically, these changes were associated with granulation tissue and eburnation of subchondral bone (Fig. 1d). Macroscopic pathology scores were 1.5-fold lower for ALM-treated knees (2.8 \pm 0.4$)$ compared to that administered saline $(4.3 \pm 1.4)$ at 4 weeks post-surgery $(P=0.018$; Fig. $1 \mathrm{e})$.

\section{Fibrotic changes in the infrapatellar fat pad}

In contrast to the normal synovial membrane and uniform distribution of adipocytes observed in IFP from non-operated knees, synovial hyperplasia $(P=0.0003$; Fig. 2a, b) and fibrous metaplasia $(P=0.002$; Fig. $2 \mathrm{a}, \mathrm{c})$ was evident in IFP from operated, saline-treated animals. However, IFP from operated knees of ALM-treated animals exhibited less extensive histopathological changes to subsynovial tissue $(P=0.033$; Fig. $2 \mathrm{a}, \mathrm{c})$ than salinetreated animals, and a trend for reduced synovitis (ns, $P=0.123$; Fig. 2a, c). Consistent with these findings, immunohistochemical analysis of the myofibroblast marker, $\alpha$-SMA demonstrated a 4.6-fold reduction in staining in IFP from ALM-treated animals, compared to those that received saline $(P=0.041$; Fig. $2 \mathrm{a}, \mathrm{d})$. While smooth muscles cells of blood vessels exhibited a strong positive reaction, positive $\alpha$-SMA staining was also evident in non-vascular cells (Fig. 2a). Similarly, a 1.4-fold reduction in collagen deposition was observed in the IFP of animals in the ALM-treatment group, compared to those that received saline $(P=0.016$; Fig. $2 \mathrm{a}, \mathrm{e})$.

\section{Fibrotic changes, ECM remodeling and inflammation in the joint capsule}

A significant increase in medial joint capsule thickness of operated knees in both saline and ALM-treated animals was observed 4 weeks after surgery $(P<0.001$; Fig. 3a, b). However, compared to animals in the saline group, mean medial joint capsule thickness in ALM-treated animals was significantly reduced (1.4-fold; $P=0.015)$. An increase in blood vessel density accompanied joint tissue hyperplasia in in knees of saline controls. These histopathological changes were ameliorated in animals that received intraarticular ALM at the time of surgery (1.5-fold decrease, $P=$ 0.02; Fig. 3a, c, d). Compared to saline-treated animals, protein and gene expression of critical fibrotic markers, $\alpha$ SMA (3.1-fold decrease, $P=0.006$ and 2.2-fold; $P=0.021$ respectively; Fig. $3 \mathrm{a}, \mathrm{d}, \mathrm{e})$ and TGF- $\beta 1$ (1.8-fold decrease, $P=0.467$ and 3.2-fold decrease, $P=0.041$; Fig. 3a, f, g), were significantly lower in anteromedial joint tissue from ALM-treated animals.

In addition to increased myofibroblast presence, the expression of key markers of tissue remodeling (MMP-3 and -13 , matrix metalloproteinases; XYLT1, xylosyltransferase- 


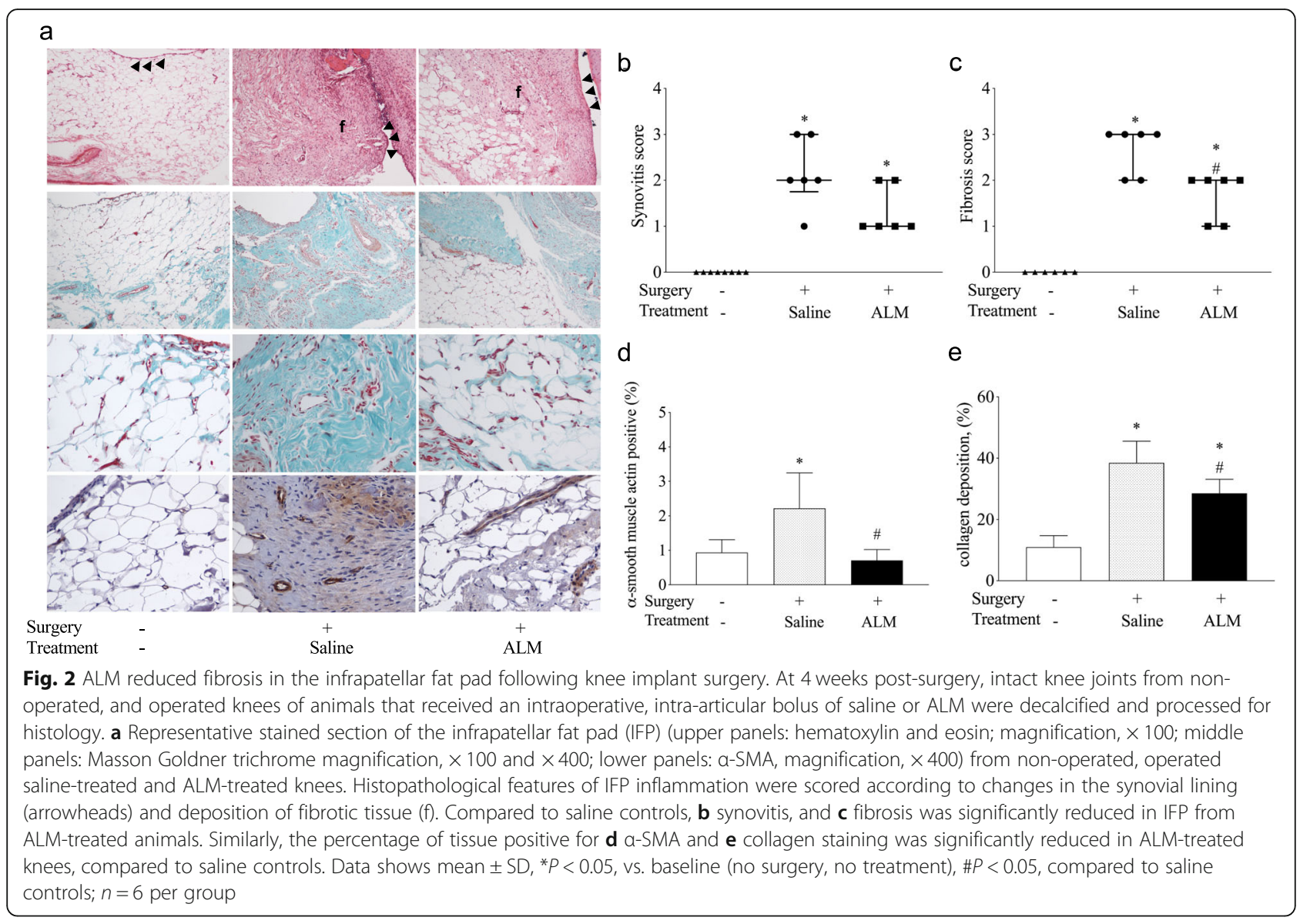

1), growth factors (CCN2, connective tissue growth factor; PDGF, platelet-derived growth factor; FGF1, fibroblast growth factor-1), and inflammatory pathway mediators (NFkB, nuclear factor kappa B; IL-6, interleukin-6) contributing to fibrosis were also measured. Compared to saline-treated animals, mRNA expression of $F g f 1$ was 2.7 fold lower $(P=0.041$; Fig. 4c) and $P d g f a 1.9$-fold lower $(P=0.031$; Fig. $4 \mathrm{~d})$ in capsular tissue from operated knees of ALM-treated animals. Though no significant differences were observed between ALM-treated and saline control operated knees for Col1a1, Col3a1, Mmp3, Mmp13, Xylt1, Ccn 2 and Il6 expression, the trend for reduction in these markers was similar to that observed for Fgf1 and Pdgfa (Fig. 4a, b; Fig. S2). Compared to salinetreated knees, expression of $N f k b$ and Nos 2 was 1.7 -fold $(P=0.034$; Fig. $4 \mathrm{e}$ ) and 1.8-fold lower (ns, $P=0.154$; Fig. 4f), respectively in ALM-treated joint capsular tissue, 4 weeks after surgery. While the concentration of IL-1 $\beta$ was significantly elevated in capsular tissue from saline-treated knees compared to non-operated knees $(P=0.043)$, levels were 2-fold lower in ALM-treated knees and comparable to those in non-operated knees (ns, $P=0.475$; Fig. 4g). Compared to non-operated knees, RANTES was elevated in the joint tissue of operated knees 4 weeks after surgery, and comparable between treatment groups $(P<0.001$ and $P<0.001$, respectively; Fig. S2). Concentrations of IL12 p70 (2-fold decrease; $P=0.077$ ) and IL-2 (4.6-fold decrease; $P=0.051$ ) also tended to be lower in joint capsule from ALM-treated animals than those that received saline, though differences were not statistically significant (Fig. 4h; Fig. S2). The additional cytokines investigated (IL- $1 \alpha$, IFN- $\gamma$, IL-13, IL-10 and IL-6) remained below the assay limit of detection in joint capsular tissue of non-operated and operated knees, 4 weeks after surgery.

\section{Systemic hematology and inflammation}

Compared to pre-surgical levels, erythrocyte number and hemoglobin were significantly reduced at day 3 post-surgery, returning to baseline levels by day 5 and consistent with a normal physiological response to surgery (Table S5). Leukocyte numbers remained comparable to baseline throughout the experimental period, with no significant differences observed between salineand ALM-treated animals (Table S5). Both the number and percentage of circulating granulocytes increased transiently within the first $24 \mathrm{~h}$ of surgery in all animals. However, compared to saline controls, the number of granulocytes was significantly higher in the ALM-treated 


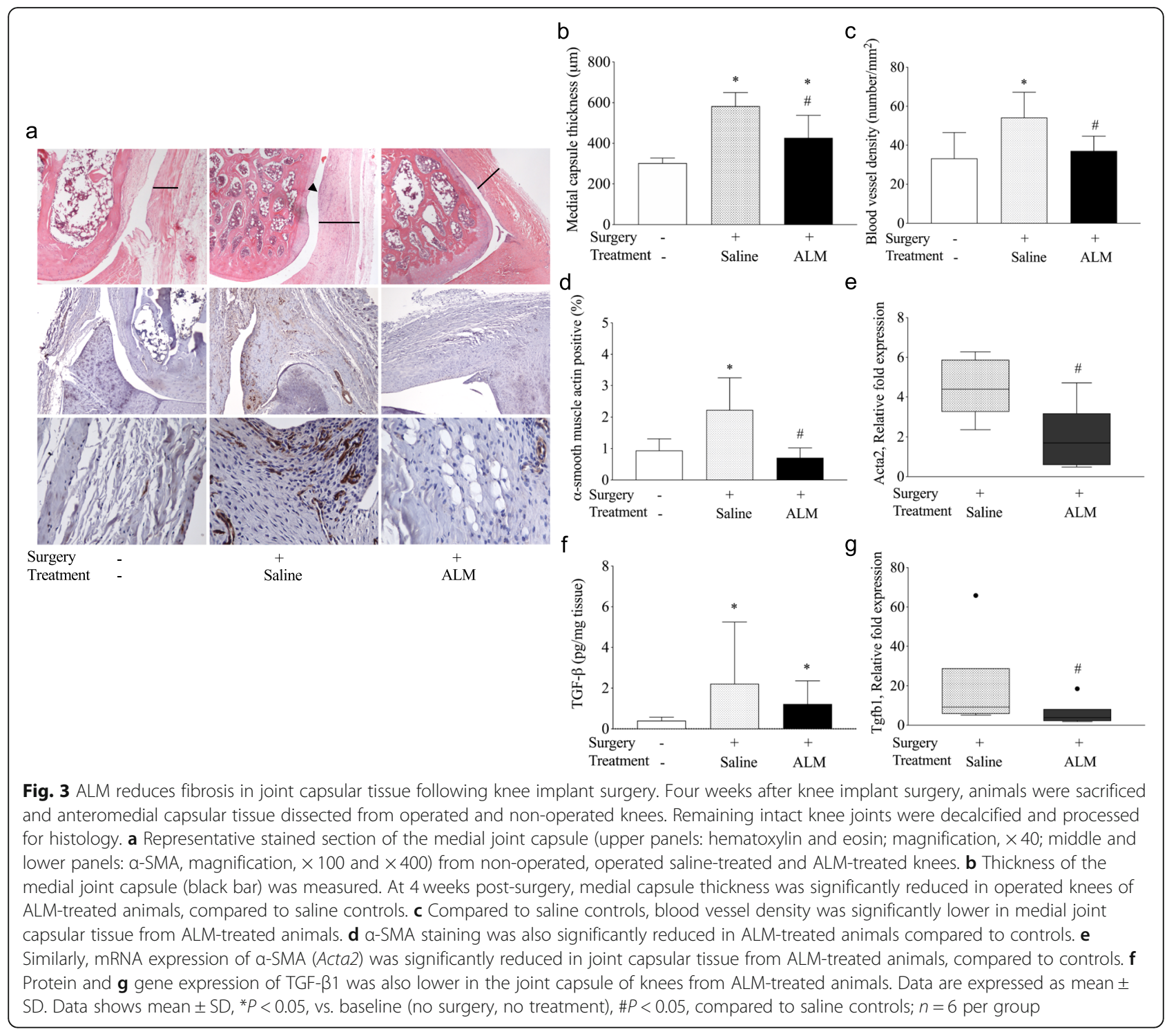

animals at $4 \mathrm{~h}(P=0.026)$ and day $3(P=0.04)$ postsurgery (Table 1; Table S5). Similarly, the number and percentage of monocytes tended to increase transiently postoperatively, with the percentage of monocytes significantly higher for ALM-treated animals $(P=0.033)$ than saline controls at day 5 (Table 1; Table S5). Consequently, while the granulocyte:monocyte ratio remained similar to preoperative levels in saline-treated controls, the ratio decreased from day 1 in ALM-treated animals and remained significantly lower than controls at day 5 following surgery (2-fold; $P=0.028$; Table 1 ). Further, due to the sustained increase in circulating monocytes in ALM-treated animals, the monocyte: lymphocyte ratio remained significantly elevated at day 5 post-surgery compared to baseline and saline controls (Table 1). Platelets remained comparable to baseline levels in ALM-treated animals following surgery. In contrast, the number of platelets increased rapidly in saline controls and were significantly higher than for ALM-treated animals at day 5 post-surgery (2.4-fold; $P=0.005$; Table S5).

Surgery-induced coagulopathy was evident in both treatment groups indicated by significantly reduced clot amplitudes (A10, MCF) and clot kinetics ( $\alpha$ angle) on EXTEM and INTEM tests at $4 \mathrm{~h}$ and day 1 , with return to baseline levels by day 3 post-surgery (Table S6). Maximum lysis was below $15 \%$ indicating no hyperfibrinolysis in response to surgery in either group.

Plasma levels of IFN- $\gamma$ and IL-6 increased significantly from baseline at 4 and $24 \mathrm{~h}$ postoperative, returning to presurgical levels within 5 days (Table 1; Table S5). A significant increase was also observed for circulating IL-10 levels at $4 \mathrm{~h}$ and $24 \mathrm{~h}$ post-surgery in saline controls and ALMtreated animals, compared to baseline. However, plasma 
a

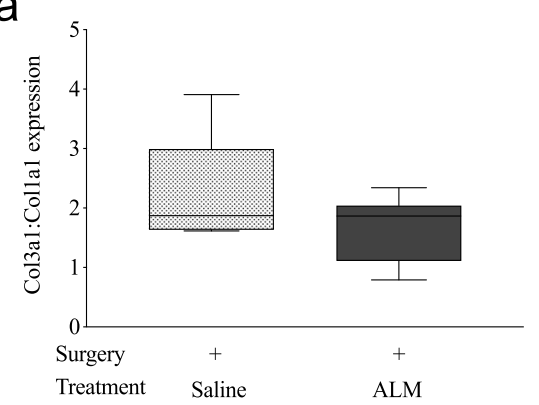

C

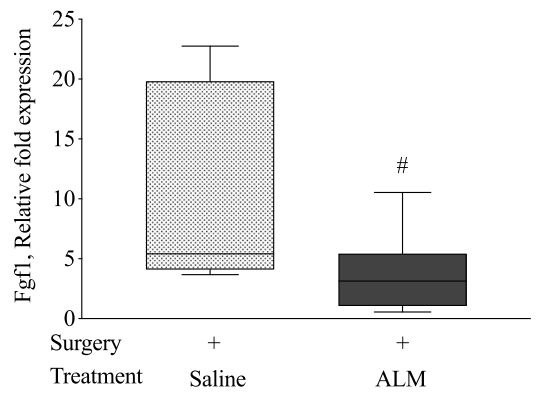

e

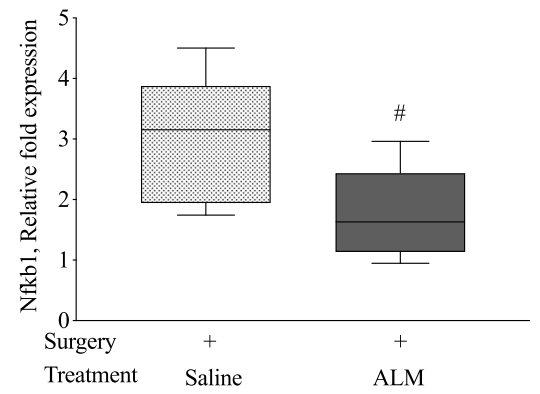

g

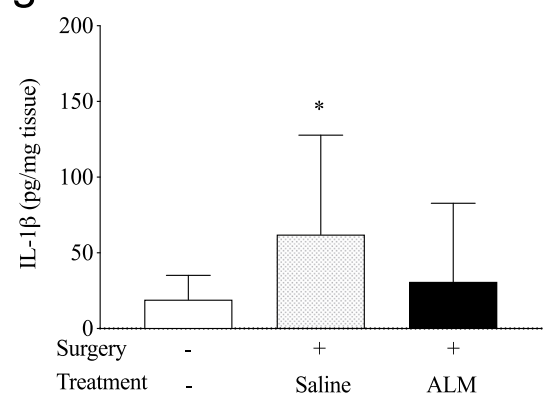

b

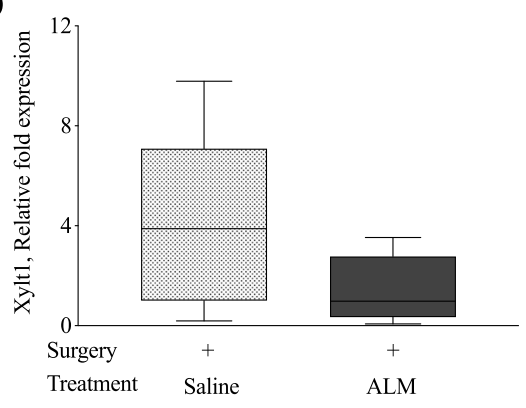

d

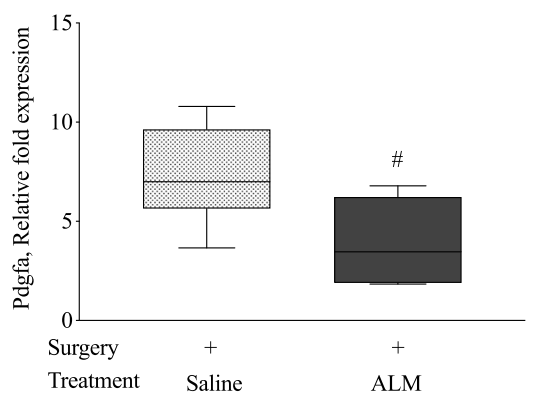

f

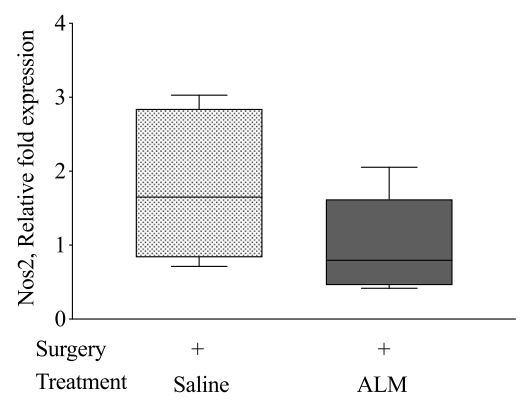

$\mathrm{h}$

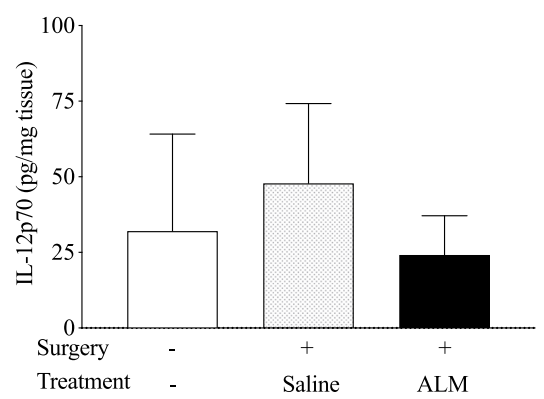

Fig. 4 Expression of key markers of ECM remodeling and inflammation in joint capsular tissue of ALM-treated and saline control animals, 4 weeks following knee implant surgery. mRNA expression of a Col3a1:Co/1a1 ratio, and $\mathbf{b}$ Xylt t tended to be lower in capsular tissue from ALM-treated animals, compared to controls, though these differences were not statistically significant. Compared to controls, c Fgf1, and $\mathbf{d}$ Pdgfa expression was significantly lower in joint tissue from ALM-treated animals. Compared to controls, mRNA expression of e NFkB1 and $\mathbf{f}$ NOS2 and protein expression of $\mathbf{g} \mathrm{LL}-1 \beta$, and $\mathbf{h} \mathrm{IL}-12 \mathrm{p} 70$ was lower in the ALM treatment group. Data are expressed as mean $\pm \mathrm{SD}$. ${ }^{*} P<0.05$, vs. baseline (no surgery, no treatment), $\# P<0.05$, compared to saline controls; $n=6$ per group 
Table 1 Hematology and systemic inflammatory responses

\begin{tabular}{|c|c|c|c|c|}
\hline Indices & Baseline & Time & Saline Control & ALM \\
\hline \multirow{5}{*}{$\begin{array}{l}\text { Granulocytes } \\
\times 10^{9} \text { cells } / \mathrm{L}\end{array}$} & \multirow[t]{5}{*}{$3.0 \pm 2.0$} & $4 \mathrm{~h}$ & $5.4 \pm 1.9$ & $8.6 \pm 2.1^{* \wedge}$ \\
\hline & & day 1 & $6.4 \pm 2.8$ & $8.3 \pm 1.2^{*}$ \\
\hline & & day 3 & $2.8 \pm 1.5$ & $4.9 \pm 1.6 \wedge$ \\
\hline & & day 5 & $2.7 \pm 1.6$ & $3.3 \pm 1.2$ \\
\hline & & day 28 & $2.1 \pm 1.3$ & $2.7 \pm 1.4$ \\
\hline \multirow{5}{*}{$\begin{array}{l}\text { Monocytes } \\
\times 10^{9} \text { cells } / \mathrm{L}\end{array}$} & \multirow[t]{5}{*}{$0.5 \pm 0.7$} & $4 \mathrm{~h}$ & $0.3 \pm 0.2$ & $0.6 \pm 0.7$ \\
\hline & & day 1 & $0.8 \pm 0.4$ & $0.8 \pm 0.3$ \\
\hline & & day 3 & $0.4 \pm 0.7$ & $1.1 \pm 1.0$ \\
\hline & & day 5 & $0.4 \pm 0.8$ & $1.1 \pm 0.8$ \\
\hline & & day 28 & $0.4 \pm 0.5$ & $0.6 \pm 0.6$ \\
\hline \multirow[t]{5}{*}{ Granulocyte:Monocyte ratio } & \multirow[t]{5}{*}{$23.4 \pm 17.7$} & $4 \mathrm{~h}$ & $41.2 \pm 35.6$ & $37.1 \pm 35.6$ \\
\hline & & day 1 & $13.8 \pm 13.0$ & $12.2 \pm 8.4$ \\
\hline & & day 3 & $30.2 \pm 22.7$ & $15.8 \pm 22.6$ \\
\hline & & day 5 & $21.2 \pm 15.8$ & $10.4 \pm 16.3^{* \wedge}$ \\
\hline & & day 28 & $22.3 \pm 20.9$ & $16.1 \pm 17.5$ \\
\hline \multirow[t]{5}{*}{ Monocyte:Lymphocyte ratio } & \multirow[t]{5}{*}{$0.06 \pm 0.09$} & $4 \mathrm{~h}$ & $0.12 \pm 0.12$ & $0.18 \pm 0.21$ \\
\hline & & day 1 & $0.22 \pm 0.1^{*}$ & $0.17 \pm 0.07$ \\
\hline & & day 3 & $0.06 \pm 0.08$ & $0.14 \pm 0.12$ \\
\hline & & day 5 & $0.06 \pm 0.08$ & $0.12 \pm 0.07^{*} \wedge$ \\
\hline & & day 28 & $0.08 \pm 0.09$ & $0.08 \pm 0.07$ \\
\hline \multirow[t]{5}{*}{$\mathrm{IL}-1 \beta, \mathrm{pg} / \mathrm{ml}$} & \multirow[t]{5}{*}{$5.7 \pm 3.8$} & $4 \mathrm{~h}$ & $8.6 \pm 7.1$ & $9.7 \pm 12.9$ \\
\hline & & day 1 & $6.1 \pm 2.5$ & $10.6 \pm 8.8$ \\
\hline & & day 3 & $3.9 \pm 2.2$ & $5.7 \pm 2.3$ \\
\hline & & day 5 & $2.9 \pm 2.4$ & $5.0 \pm 5.3$ \\
\hline & & day 28 & $17.0 \pm 12.9^{*}$ & $7.4 \pm 4.2^{\wedge}$ \\
\hline \multirow[t]{5}{*}{$\mathrm{IFN}-\gamma, \mathrm{pg} / \mathrm{ml}$} & \multirow[t]{5}{*}{ n.d. ${ }^{a}$} & $4 \mathrm{~h}$ & $52.5 \pm 24.6^{*}$ & $32.7 \pm 21.3^{*}$ \\
\hline & & day 1 & $43.2 \pm 31.6^{*}$ & n.d. \\
\hline & & day 3 & $33.1 \pm 24.4$ & n.d. \\
\hline & & day 5 & $24.7 \pm 5.6$ & $23.6 \pm 2.0$ \\
\hline & & day 28 & n.d. & n.d. \\
\hline \multirow[t]{5}{*}{ IL-10, pg/ml } & \multirow[t]{5}{*}{$16.3 \pm 10.3$} & $4 \mathrm{~h}$ & $60.4 \pm 30.0^{*}$ & $67.1 \pm 54.9^{*}$ \\
\hline & & day 1 & $37.4 \pm 12.4^{*}$ & $46.4 \pm 30.9^{*}$ \\
\hline & & day 3 & $40.1 \pm 15.7^{*}$ & $42.7 \pm 12.8^{*}$ \\
\hline & & day 5 & $32.0 \pm 9.9^{*}$ & $21.7 \pm 15.0 \wedge$ \\
\hline & & day 28 & $53.7 \pm 33.9^{*}$ & $25.0 \pm 14.2^{\wedge}$ \\
\hline
\end{tabular}

$4 \mathrm{~h}$, day 1 and 3, $n=6$; day 5 and 28, $n=12$. Baseline values were obtained from tail vein of 8 healthy anesthetised rats (refer Methods). Data represent mean \pm SD n.d. not detected

${ }^{*} P<0.05$ compared to baseline. $\wedge P<0.05$ compared to saline. ${ }^{a}$ limit of detection IFN- $\gamma, 15.3 \mathrm{pg} / \mathrm{ml}$

IL-10 levels remained significantly higher in animals that received saline alone at day 5 , and 4 weeks post-surgery, compared to those administered ALM $(P=0.008$ and $P=$ 0.005 , respectively; Table 1$)$. Similarly, levels of the proinflammatory cytokine IL-1 $\beta$ were significantly elevated in plasma from saline controls compared to animals administered ALM at 4 weeks post-surgery $(P=0.025$; Table 1$)$. No significant changes were observed in plasma concentrations of MCP-1, TNF- $\alpha$, IL-12p70, or IL-4 following surgery, with levels comparable for saline controls and ALM-treated animals at all timepoints investigated (Table S5).

\section{Endothelial activation}

To compare the extent of vascular injury in ALM- and saline-treated animals, four soluble cellular adhesion molecules (sICAM, sE-selectin, adiponectin and vWF) 
were measured in plasma at $4 \mathrm{~h}$ and day 1 postsurgery (Table S5). sICAM levels remained comparable to preoperative ranges. In contrast, concentrations of sE-selectin, adiponectin and vWF increased significantly from baseline within $4 \mathrm{~h}$ of surgery. However, no differences were observed in concentrations of these vascular injury markers between saline and ALM-treated animals at either time point (Table S5).

\section{Discussion}

Acquired idiopathic stiffness and arthrofibrosis following primary TKA are common causes of reduced ROM and postoperative pain, often requiring extended rehabilitation programs and/or repeat surgical correction [3]. In this study, we report that IA administration of ALM at the end of knee implant surgery in the rat appears to increase ROM and to improve healing after 4 weeks, with the macroscopic restoration of healthy joint tissue architecture. We also showed that ALM significantly decreased fibrotic markers within synovial and capsular tissue of the implanted knee, compared to controls. These fibrotic changes paralleled a reduction in systemic inflammation immediately following, and joint tissue inflammation over the four-week postoperative period.

\section{ALM therapy reduces fibrotic changes in the infrapatellar fat pad}

The finding that ALM significantly reduced the expression of $\alpha$-SMA, a marker for myofibroblasts which are the critical cell mediators of fibrosis [22], suggests that the drug therapy may have reduced fibrosis in the IFP in our TKA model. The histological assessment of the IFP further supports this (Fig. 2). Although the IFP is commonly removed by surgeons to assist with the alignment and fitting of the artificial knee components and reduce postoperative anterior knee pain [23], others argue that its' removal is unnecessary [24]. Given that the IFP is highly vascularized at the periphery and richly innervated with nerves and lymphatic vessels, it plays a significant role in local and systemic immune responses to surgery. For example, the IFP contains adipocytes, preadipocytes, macrophages, fibroblasts, and other cells that secrete cytokines, adipokines, and other neural, inflammatory and metabolic mediators [25] that may have protective functions beyond the joint [26]. Given that there is little experimental support that removal of the IFP reduces postoperative pain [24], ALM-induced reduction of inflammation and fibrosis may have wider significance for IFP preservation during TKA and may confer immune benefits for the patient [27]. Protecting the IFP with ALM may also have utility in minimally invasive arthroscopic procedures such as meniscectomy and ACL reconstruction.
ALM therapy reduces fibrosis, ECM remodeling and inflammation in the joint capsule

Our study also showed that ALM protection extended to the joint capsule itself. ALM protection manifested as significant decreases in expression of TGF- $\beta$, FGF1, PDGFA and $\alpha$-SMA in joint tissue, and tendency for lower expression of XT-1 and CCN2, compared to saline controls (Figs. 3, 4). Histologically, joint tissue from ALM-treated animals exhibited significant reductions in synovitis, myofibroblasts, proteoglycan accumulation and blood vessel density, and more closely resembled healthy joint capsule architecture than controls. Suppression of inflammation in ALM-treated knees, was also supported by decreased levels of key immune mediators IL-1 $\beta$, IL-12 and iNOS (nitric oxide production) in capsular tissue (Fig. 4). Of particular importance to creating a more permissive healing environment, ALM markedly reduced expression of $\mathrm{NFkB}$, a prime driver of inflammation and fibrosis [2], compared to controls. Currently, we do not know if ALM directly inhibits the NFKB signaling pathway, or exerts indirect effects via upstream mediators.

\section{ALM alters early postoperative systemic inflammatory responses}

Granulocyte and monocytes are key effector cells of the innate immune response. The transient increase in granulocytes and monocytes observed in all animals at $4 \mathrm{~h}$ and day 1 postoperative (Table 1 ) is consistent with the systemic inflammatory response to anesthesia and surgical trauma $[8,10,12,16,28,29]$. Within minutes to hours of tissue trauma, granulocytes (mostly neutrophils) are rapidly mobilized from the circulation and bone marrow to local sites, followed later by monocytes [28]. Together with tissue-resident cells, they are key orchestrators of the cellular and molecular events of the acute inflammatory response required to mitigate further tissue damage and facilitate healing. Following surgery, we showed that circulating total leukocyte numbers were comparable for both treatment groups (Table S5). However, in ALM-treated animals we found significant differences in the proportion of granulocytes and monocytes over the first 5 days compared to controls (Table 1). In the first 3 days following surgery, there was a spike in granulocytes in the ALM group followed by a steady rise in monocytes to day 5 . In contrast, circulating monocyte numbers remained comparable to preoperative levels in saline controls over the same period (Table 1, Table S5). This is also reflected in the significantly lower granulocyte:monocyte ratio at day 5 in ALM animals (Table 1). This time-dependent progression of neutrophils and monocytes can profoundly impact the duration and magnitude of subsequent inflammatory, immune and tissue repair responses [28, 30]. 
While we did not measure immune cells at the injury site, the data indicate that ALM may act as an inflammatory brake, in turn leading to reduced fibrosis and improved joint tissue healing. This hypothesis is further supported by control animals showing persistent inflammation (Table 1, Fig. 4) systemically and within joint tissues of the implanted knee at 4 weeks postoperative. Also, surgery-induced IL-10 plasma concentrations, which are associated with decreased long-term functional performance in the operated limb following TKA [31], were also lower in ALM-treated animals (Table 1). Further investigations are warranted to elucidate the molecular and cellular mechanisms underpinning the effect of ALM on inflammation resolution within the local microenvironment of the operated knee in the early postoperative period.

\section{Limitations of the study}

A potential limitation of the rat model used in the present study is that it may not reflect some aspects of the tissue trauma evoked following TKA [1]. Patients undergoing TKA will already have pre-existing trauma and inflammation from osteoarthritis and most have multiple comorbidities, which may affect the duration of the operation, and extent of inflammation and recovery $[1,32]$. The rat TKA model however, does elicit a strong trauma-induced inflammatory response leading to joint fibrosis, and is useful for studying biochemical and histological changes underpinning recovery dynamics [16]. Although we demonstrated an association between early inflammatory responses and 4-week pathology, in future studies it would be essential to investigate the changes in joint tissue inflammation and immune cell infiltration within the first week of knee implant surgery. Western blot analysis could also be performed to support the mRNA expression and histological data. Further studies will also employ microCT to investigate the effect of ALM on osseointegration of TKA prostheses. Overall, while not reproducing the damaged knee of the TKA patient, the rat model of knee implant surgery is a useful model to study the effect of new drugs and underlying mechanisms controlling inflammation as part of their translation to improve outcomes in humans.

\section{Clinical relevance}

TKA is one of the most commonly performed inpatient surgical procedures globally, and it is estimated to reach 3.48 million procedures in the USA annually by 2030 [32].

Arthrofibrosis is a common perioperative complication with debilitating consequences $[1,2]$. Our study shows ALM administration into the joint after capsule closure, and before skin closure has the potential to reduce inflammation, stiffness and arthrofibrosis in the TKA patient. To date, alternative immunomodulatory candidates for arthrofibrosis require sustained or repeated drug administration to reduce fibrotic tissue within the knee [33, 34]. An advantage of ALM therapy is that a single local injection into the joint is required for its benefit. This benefit cannot be afforded by the individual actives $\mathrm{A}, \mathrm{L}$ or $\mathrm{M}$ alone; rather, it is the combination that reduces inflammation, coagulopathy, and ischemiareperfusion injury $[8,10-14,35,36]$. What is most interesting, is that the circulating half-lives of $\mathrm{A}, \mathrm{L}$ and $\mathrm{M}$ are seconds to hours, yet in the present study we show the benefit can last 4 weeks, indicating that ALM is acting early to 'switch' to an improved healing phenotype [11, 14]. Future studies will elucidate the molecular pathways underlying ALMs anti-inflammatory, pro-resolving, and anti-fibrotic properties before translation into humans.

\section{Conclusions}

In conclusion, the present study demonstrates that IA administration of a single ALM bolus before skin closure alleviated post-TKA knee fibrosis in a rat model. ALM therapy appears to modulate inflammatory mediators in the early postoperative period, favoring tissue repair rather than fibrosis. Our findings provide support for further evaluation of ALM therapy as an upfront strategy for improved healing and recovery after TKA, and possibly other orthopedic procedures.

\section{Supplementary Information}

The online version contains supplementary material available at https://doi. org/10.1186/s41231-021-00084-3.

Additional file 1: Fig. S1. Schematic representation of the study protocol for the evaluation of ALM therapy in a rat model of knee replacement surgery.

Additional file 2: Table S1. Surgery details: time of surgery, blood loss, anesthesia time.

Additional file 3: Table S2. Macroscopic scoring scheme. Additional file 4: Table S3. Primers used for quantitative real-time PCR analysis.

Additional file 5: Table S4. Histopathology grading scheme.

Additional file 6: Table S5. Postoperative clinical, hematology and systemic inflammatory parameters.

Additional file 7: Table S6. ROTEM parameters.

Additional file 8: Fig. S2. Expression of inflammatory and ECM remodeling markers in joint capsular tissue.

\section{Abbreviations}

ACL: Anterior cruciate ligament; ALM: Adenosine, lidocaine and $\mathrm{Mg}^{2+}$; $\mathrm{a}^{-}$ SMA: Alpha smooth muscle actin; CCN2: Connective tissue growth factor: ECM: Extracellular matrix; EDTA: Ethylenediaminetetraacetic acid;

FGF: Fibroblast growth factor; H\&E: Hematoxylin and eosin; IA: Intra-articular; IFP: Infrapatellar fat pad; IL: Interleukin; MMP: Matrix metalloproteinase; NFkB: Nuclear factor-kappa b; OARSI: Osteoarthritis Research Society International; PFA: Paraformaldehyde; PDGFA: Platelet-derived growth factor subunit A; RANTES: Regulated upon activation, normal T cell expressed and presumably secreted; RNA: Ribonucleic acid; ROM: Range of motion; ROTEM: Rotational thromboelastometry; SD: Standard deviation; 
sICAM: Soluble intercellular adhesion molecule; TGF $\beta$ : Transforming growth factor beta; TKA: Total knee arthroplasty; UHXLPE: Ultra highly cross-linked polyethylene; VWF: Von Willebrand factor; XYLT: Xylosyltransferase

\section{Acknowledgements}

We thank Mr. Rob Wood (Stryker Orthopaedics) and Mr. lain McMillan (Enztec Pty Ltd) for their kind provision of customized titanium and UHXLPE implants. We are grateful to Mrs. Lindy McEwen, Ms. Regina Kirk, Dr. Ben Brandon, Dr. Ivana-Aleksandra Jovanovic, Mr. Rhys Gillman and Ms. Andrea Grant for assistance with animal surgeries and sample collection.

\section{Authors' contributions}

All authors conceived the study and participated in study design. JM, HL, EB, CD and PM contributed to data collection and analysis. JM, HL and GPD prepared the draft manuscript. All authors critically reviewed and approved the final manuscript.

\section{Funding}

This research was supported by the Australian Orthopaedic Association, the Townsville Hospital and Health Services Study, Education, Research and Training Fund, and internal funds of the Orthopaedic Research Institute of Queensland and James Cook University.

\section{Availability of data and materials}

Not applicable.

\section{Ethics approval and consent to participate}

The Institutional Animal Ethics Committee approved the experimental protocol (approval no. A2487). The study was conducted in accordance with the ARRIVE guidelines and the NHMRC guidelines for the care and use of laboratory animals.

\section{Consent for publication}

Not applicable.

\section{Competing interests}

GPD is the sole inventor of the ALM concept for cardioplegia and trauma, but has no financial interests in any entity or business. All other authors have no competing interests to declare.

\section{Author details}

'Orthopaedic Research Institute of Queensland, Townsville, Queensland 4812, Australia. ${ }^{2}$ Heart, Trauma and Sepsis Research Laboratory, College of Medicine and Dentistry, James Cook University, Townsville, Queensland 4811, Australia.

\section{Received: 7 October 2020 Accepted: 19 January 2021} Published online: 01 February 2021

\section{References}

1. Thompson R, Novikov D, Cizmic Z, Feng JE, Fideler K, Sayeed Z, et al. Arthrofibrosis after total knee arthroplasty: pathophysiology, diagnosis, and management. Orthop Clinics. 2019;50(3):269-79.

2. Usher KM, Zhu S, Mavropalias G, Carrino JA, Zhao J, Xu J. Pathological mechanisms and therapeutic outlooks for arthrofibrosis. Bone Res. 2019;7(1):9.

3. Tibbo ME, Limberg AK, Salib CG, Ahmed AT, van Wijnen AJ, Berry DJ, et al. Acquired idiopathic stiffness after total knee arthroplasty: a systematic review and meta-analysis. J Bone Joint Surg Am. 2019;101(14):1320-30.

4. Abdul N, Dixon D, Walker A, Horabin J, Smith N, Weir DJ, et al. Fibrosis is a common outcome following total knee arthroplasty. Sci Rep. 2015;5(1): 16469.

5. Chen AF, Lee YS, Seid AJ, Abboud JA. Arthrofibrosis and large joint scarring. Connect Tissue Res. 2019;60(1):21-8.

6. Ekhtiari S, Horner NS, de Sa D, Simunovic N, Hirschmann MT, Ogilvie R, et al. Arthrofibrosis after ACL reconstruction is best treated in a step-wise approach with early recognition and intervention: a systematic review. Knee Surg Sports Traumatol Arthrosc. 2017;25(12):3929-37.

7. Kiapour AM, Murray MM. Basic science of anterior cruciate ligament injury and repair. Bone Joint Res. 2014;3(2):20-31.
8. Davenport L, Letson HL, Dobson GP. Immune-inflammatory activation after a single laparotomy in a rat model: effect of adenosine, lidocaine and Mg2+ infusion to dampen the stress response. Innate Immun. 2017;23(5):482-94.

9. Dobson G, Letson H. A new ultra-small volume fluid for far-forward, noncompressible hemorrhage and traumatic brain injury. Maryland: Fort Detrick; 2016. Report No.: W81XWH-15-1-0002

10. Dobson GP. Trauma of major surgery: a global problem that is not going away. Int J Surg. 2020;81:47-54

11. Dobson GP, Letson HL. Far forward gaps in hemorrhagic shock and prolonged field care: an update of ALM fluid therapy for field use. J Spec Oper Med. 2020; In Press (Accepted for Fall Issue).

12. Letson H, Dobson G. Adenosine, lidocaine and Mg2+ (ALM) fluid therapy attenuates systemic inflammation, platelet dysfunction and coagulopathy after non-compressible truncal hemorrhage. PLOS One. 2017;12(11):e0188144.

13. Letson $\mathrm{HL}$, Dobson GP. $3 \% \mathrm{NaCl}$ adenosine, lidocaine, Mg2+ (ALM) bolus and 4 hours "drip" infusion reduces noncompressible hemorrhage by $60 \%$ in a rat model. J Trauma Acute Care Surg. 2017;82(6):1063-72.

14. Letson HL, Morris JL, Biros E, Dobson GP. Adenosine, lidocaine, and Mg2+ fluid therapy leads to 72-hour survival after hemorrhagic shock: a model for studying differential gene expression and extending biological time. J Trauma Acute Care Surg. 2019;87(3):606-13.

15. McCutchan A, Dobson GP, Stewart N, Letson HL, Grant AL, Jovanovic I-A, et al. Absence of cytotoxic and inflammatory effects following in vitro exposure of chondrogenically-differentiated human mesenchymal stem cells to adenosine, lidocaine and Mg2+ solution. J Exp Orthop. 2019;6(1):16.

16. Morris JL, Letson HL, Grant A, Wilkinson M, Hazratwala K, McEwen P. Experimental model of peri-prosthetic infection of the knee caused by Staphylococcus aureus using biomaterials representative of modern TKA. Biol Open. 2019;8(9):bio045203.

17. Gao Z-Y, Wu J-X, Liu W-B, Sun J-K. Reduction of adhesion formation after knee surgery in a rat model by botulinum toxin A. Biosci Rep. 2017;37(2): BSR20160460.

18. Af Klint E, Catrina Al, Matt $P$, Neregråd P, Lampa J, Ulfgren A-K, et al. Evaluation of arthroscopy and macroscopic scoring. Arthritis Res Ther. 2009; 11(3):R81.

19. Gerwin N, Bendele AM, Glasson S, Carlson CS. The OARSI histopathology initiative - recommendations for histological assessments of osteoarthritis in the rat. Osteoarthr Cartil. 2010;18:S24-34.

20. Takahashi I, Matsuzaki T, Kuroki H, Hoso M. Induction of osteoarthritis by injecting monosodium iodoacetate into the patellofemoral joint of an experimental rat model. PLoS One. 2018;13(4):e0196625.

21. Kitagawa T, Nakase J, Takata Y, Shimozaki K, Asai K, Tsuchiya H. Histopathological study of the infrapatellar fat pad in the rat model of patellar tendinopathy: a basic study. Knee. 2019;26(1):14-9.

22. Wynn TA. Cellular and molecular mechanisms of fibrosis. J Pathol. 2008; 214(2):199-210.

23. Ryan TJ. Infrapatellar fat pad resection during total knee arthroplasty: yet another reason for? Ann Rheum Dis. 2019;78(7):e63.

24. Nisar S, Lamb JN, Somashekar N, Pandit H, van Duren BH. Preservation vs. resection of the infrapatellar fat pad during total knee arthroplasty part II: a systematic review of published evidence. Knee. 2019;26(2):422-6.

25. Jiang LF, Fang $J H$, Wu LD. Role of infrapatellar fat pad in pathological process of knee osteoarthritis: future applications in treatment. World J Clin Cases. 2019;7(16):2134-42.

26. Morris $\mathrm{L}$, Letson $\mathrm{HL}$, Gillman $\mathrm{R}$, Hazratwala $\mathrm{K}$, Wilkinson M, McEwen $\mathrm{P}$, et al. The CNS theory of osteoarthritis: opportunities beyond the joint. Semin Arthritis Rheum. 2019:49(3):331-6.

27. Grant RW, Dixit VD. Adipose tissue as an immunological organ. Obesity (Silver Spring). 2015;23(3):512-8.

28. Prame Kumar K, Nicholls AJ, Wong CHY. Partners in crime: neutrophils and monocytes/macrophages in inflammation and disease. Cell Tissue Res. 2018; 371(3):551-65.

29. Alhayyan A, McSorley S, Roxburgh C, Kearns R, Horgan P, McMillan D. The effect of anesthesia on the postoperative inflammatory response in patients undergoing surgery: a systematic review and meta-analysis. Surg Open Sci. 2020;2:1-21.

30. Huber-Lang M, Lambris JD, Ward PA. Innate immune responses to trauma. Nat Immunol. 2018;19(4):327-41.

31. Langkilde A, Jakobsen TL, Bandholm TQ, Eugen-Olsen J, Blauenfeldt T, Petersen J, et al. Inflammation and postoperative recovery in patients 
undergoing total knee arthroplasty-secondary analysis of a randomized controlled trial. Osteoarthr Cartil. 2017;25(8):1265-73.

32. Feng JE, Novikov D, Anoushiravani AA, Schwarzkopf R. Total knee arthroplasty: improving outcomes with a multidisciplinary approach. J Multidiscip Healthc. 2018;11:63-73.

33. Atluri K, Brouillette MJ, Seol D, Khorsand B, Sander E, Salem AK, et al. Sulfasalazine resolves joint stiffness in a rabbit model of arthrofibrosis. J Orthop Res. 2020;38(3):629-38.

34. Salib CG, Reina N, Trousdale WH, Limberg AK, Tibbo ME, Jay AG, et al. Inhibition of COX-2 pathway as a potential prophylaxis against arthrofibrogenesis in a rabbit model of joint contracture. J Orthop Res. 2019; 37(12):2609-20.

35. Dobson GP, Letson HL. Adenosine, lidocaine, and Mg2+ (ALM): From cardiac surgery to combat casualty care-Teaching old drugs new tricks. J Trauma Acute Care Surg. 2016;80(1):135-45.

36. Dobson GP, Morris JL, Davenport L, Letson HL. Traumatic-induced coagulopathy as a systems failure: a new window into hemostasis. Semin Thromb Hemost. 2020;46(2):199-214.

\section{Publisher's Note}

Springer Nature remains neutral with regard to jurisdictional claims in published maps and institutional affiliations.

Ready to submit your research? Choose BMC and benefit from:

- fast, convenient online submission

- thorough peer review by experienced researchers in your field

- rapid publication on acceptance

- support for research data, including large and complex data types

- gold Open Access which fosters wider collaboration and increased citations

- maximum visibility for your research: over $100 \mathrm{M}$ website views per year

At BMC, research is always in progress.

Learn more biomedcentral.com/submissions 